\title{
A NOTE ON ASYMPTOTIC STABILITY CONDITIONS FOR DELAY DIFFERENCE EQUATIONS
}

\author{
T. KAEWONG, Y. LENBURY, AND P. NIAMSUP
}

Received 26 May 2004 and in revised form 27 February 2005

We obtain necessary and sufficient conditions for the asymptotic stability of the linear delay difference equation $x_{n+1}+p \sum_{j=1}^{N} x_{n-k+(j-1) l}=0$, where $n=0,1,2, \ldots, p$ is a real number, and $k, l$, and $N$ are positive integers such that $k>(N-1) l$.

\section{Introduction}

In [4], the asymptotic stability condition of the linear delay difference equation

$$
x_{n+1}-x_{n}+p \sum_{j=1}^{N} x_{n-k+(j-1) l}=0,
$$

where $n \in \mathbb{N}_{0}=\mathbb{N} \cup\{0\}, p$ is a real number, and $k, l$, and $N$ are positive integers with $k>(N-1) l$ is given as follows.

Theorem 1.1. Let $k, l$, and $N$ be positive integers with $k>(N-1) l$. Then the zero solution of (1.1) is asymptotically stable if and only if

$$
0<p<\frac{2 \sin (\pi / 2 M) \sin (l \pi / 2 M)}{\sin (N l \pi / 2 M)}
$$

where $M=2 k+1-(N-1) l$.

Theorem 1.1 generalizes asymptotic stability conditions given in [1, page 87], [2, 3, 5], and [6, page 65]. In this paper, we are interested in the situation when (1.1) does not depend on $x_{n}$, namely we are interested in the asymptotic stability of the linear delay difference equation of the form

$$
x_{n+1}+p \sum_{j=1}^{N} x_{n-k+(j-1) l}=0,
$$

where $n \in \mathbb{N}_{0}=\mathbb{N} \cup\{0\}, p$ is a real number, and $k, l$, and $N$ are positive integers with $k \geq(N-1) l$. Our main theorem is the following. 
Theorem 1.2. Let $k, l$, and $N$ be positive integers with $k \geq(N-1) l$. Then the zero solution of (1.3) is asymptotically stable if and only if

$$
-\frac{1}{N}<p<p_{\min }
$$

where $p_{\min }$ is the smallest positive real value of $p$ for which the characteristic equation of (1.3) has a root on the unit circle.

\section{Proof of theorem}

The characteristic equation of (1.3) is given by

$$
F(z)=z^{k+1}+p\left(z^{(N-1) l}+\cdots+z^{l}+1\right)=0 .
$$

For $p=0, F(z)$ has exactly one root at 0 of multiplicity $k+1$. We first consider the location of the roots of (2.1) as $p$ varies. Throughout the paper, we denote the unit circle by $C$ and let $M=2 k+2-(N-1) l$.

Proposition 2.1. Let $z$ be a root of (2.1) which lies on $C$. Then the roots $z$ and $p$ are of the form

$$
\begin{gathered}
z=e^{w_{m} i}, \\
p=(-1)^{m+1} \frac{\sin \left(l w_{m} / 2\right)}{\sin \left(N l w_{m} / 2\right)} \equiv p_{m}
\end{gathered}
$$

for some $m=0,1, \ldots, M-1$, where $w_{m}=(2 m / M) \pi$. Conversely, if $p$ is given by (2.3), then $z=e^{w_{m} i}$ is a root of $(2 \cdot 1)$.

Proof. Note that $z=1$ is a root of (2.1) if and only if $p=-1 / N$, which agrees with (2.2) and (2.3) for $w_{m}=0$. We now consider the roots of (2.1) which lie on $C$ except the root $z=$ 1. Suppose that the value $z$ satisfies $z^{N l}=1$ and $z^{l} \neq 1$. Then $z^{N l}-1=\left(z^{l}-1\right)\left(z^{(N-1) l}+\right.$ $\left.\cdots+z^{l}+1\right)=0$ which gives $z^{(N-1) l}+\cdots+z^{l}+1=0$, and hence $z$ is not a root of $(2.1)$. As a result, to determine the roots of (2.1) which lie on $C$, it suffices to consider only the value $z$ such that $z^{N l} \neq 1$ or $z^{l}=1$. For these values of $z$, we may write (2.1) as

$$
p=-\frac{z^{k+1}}{z^{(N-1) l}+\cdots+z^{l}+1} .
$$

Since $p$ is real, we have

$$
p=-\frac{\bar{z}^{k+1}}{\bar{z}^{(N-1) l}+\cdots+\bar{z}^{l}+1}=-\frac{z^{-k-1+(N-1) l}}{z^{(N-1) l}+\cdots+z^{l}+1},
$$

where $\bar{z}$ denotes the conjugate of $z$. It follows from (2.4) and (2.5) that

$$
z^{2 k+2-(N-1) l}=1
$$

which implies that (2.2) is valid for $m=0,1, \ldots, M-1$ except for those integers $m$ such that $e^{N l w_{m} i}=1$ and $e^{l w_{m} i} \neq 1$. We now show that $p$ is of the form stated in (2.3). There are two cases to be considered as follows. 
Case 1. $z$ is of the form $e^{w_{m} i}$ for some $m=1,2, \ldots, M-1$ and $z^{N l} \neq 1$.

From (2.4), we have

$$
\begin{aligned}
p & =-\frac{z^{k+1}\left(z^{l}-1\right)}{z^{N l}-1}=-\frac{e^{(k+1) w_{m} i}\left(e^{l w_{m} i}-1\right)}{e^{N l w_{m} i}-1} \\
& =-\frac{e^{(k+1-(N-1)(l / 2)) w_{m} i}\left(e^{l w_{m} i / 2}-e^{-l w_{m} i / 2}\right)}{e^{N l w_{m} i / 2}-e^{-N l w_{m} i / 2}} \\
& =-e^{(k+1-(N-1)(l / 2)) w_{m} i} \frac{\sin \left(l w_{m} / 2\right)}{\sin \left(N l w_{m} / 2\right)} \\
& =-e^{m \pi i} \frac{\sin \left(l w_{m} / 2\right)}{\sin \left(N l w_{m} / 2\right)}=(-1)^{m+1} \frac{\sin \left(l w_{m} / 2\right)}{\sin \left(N l w_{m} / 2\right)} \equiv p_{m} .
\end{aligned}
$$

Case 2. $z$ is of the form $e^{w_{m} i}$ for some $m=1,2, \ldots, M-1$ and $z^{l}=1$.

In this case, we have $l w_{m}=2 q \pi$ for some positive integer $q$. Then taking the limit of $p_{m}$ as $l w_{m} \rightarrow 2 q \pi$, we obtain

$$
p=-\frac{(-1)^{m+q(N-1)}}{N}
$$

From these two cases, we conclude that $p$ is of the form in (2.3) for $m=1,2, \ldots, M-1$ except for those $m$ such that $e^{N l w_{m} i}=1$ and $e^{l w_{m} i} \neq 1$.

Conversely, if $p$ is given by (2.3), then it is obvious that $z=e^{w_{m} i}$ is a root of (2.1). This completes the proof of the proposition.

From Proposition 2.1, we may consider $p$ as a holomorphic function of $z$ in a neighborhood of each $z_{m}$. In other words, in a neighborhood of each $z_{m}$, we may consider $p$ as a holomorphic function of $z$ given by

$$
p(z)=-\frac{z^{k+1}}{z^{(N-1) l}+\cdots+z^{l}+1} .
$$

Then we have

$$
\frac{d p(z)}{d z}=-\frac{(k+1) z^{k}}{z^{(N-1) l}+\cdots+z^{l}+1}+\frac{z^{k}\left\{(N-1) l z^{(N-1) l}+\cdots+l z^{l}\right\}}{\left(z^{(N-1) l}+\cdots+z^{l}+1\right)^{2}} .
$$

From this, we have the following lemma.

Lemma 2.2. $d p /\left.d z\right|_{z=e^{w_{m}} i} \neq 0$. In particular, the roots of (2.1) which lie on $C$ are simple.

Proof. Suppose on the contrary that $d p /\left.d z\right|_{z=e^{w_{m} i}}=0$. We divide (2.10) by $p(z) / z$ to obtain

$$
k+1-\frac{l\left\{(N-1) z^{(N-1) l}+\cdots+z^{l}\right\}}{z^{(N-1) l}+\cdots+z^{l}+1}=0 .
$$

Substituting $z$ by $1 / \bar{z}$ in $(2.10)$, we obtain

$$
k+1-\frac{l\left\{(N-1)+(N-2) z^{l}+\cdots+z^{(N-2) l}\right\}}{z^{(N-1) l}+\cdots+z^{l}+1}=0 .
$$




\section{A note on asymptotic stability conditions}

By adding (2.11) and (2.12), we obtain

$$
2 k+2-(N-1) l=0
$$

which contradicts $k \geq(N-1) l$. This completes the proof.

From Lemma 2.2, there exists a neighborhood of $z=e^{w_{m} i}$ such that the mapping $p(z)$ is one to one and the inverse of $p(z)$ exists locally. Now, let $z$ be expressed as $z=r e^{i \theta}$. Then we have

$$
\frac{d z}{d p}=\frac{z}{r}\left\{\frac{d r}{d p}+i r \frac{d \theta}{d p}\right\}
$$

which implies that

$$
\frac{d r}{d p}=\operatorname{Re}\left\{\frac{r}{z} \frac{d z}{d p}\right\}
$$

as $p$ varies and remains real. The following result describes the behavior of the roots of (2.1) as $p$ varies.

Proposition 2.3. The moduli of the roots of (2.1) at $z=e^{w_{m} i}$ increase as $|p|$ increases.

Proof. Let $r$ be the modulus of $z$. Let $z=e^{w_{m} i}$ be a root of (2.1) on C. To prove this proposition, it suffices to show that

$$
\left.\frac{d r}{d p} \cdot p\right|_{z=e^{w_{m}}}>0
$$

There are two cases to be considered.

Case $1\left(z^{N l} \neq 1\right)$. In this case, we have

$$
p(z)=-\frac{z^{k+1}\left(z^{l}-1\right)}{z^{N l}-1}=-\frac{z^{k} f(z)}{z^{N l}-1}
$$

where $f(z)=z\left(z^{l}-1\right)$. Then

$$
\frac{d p}{d z}=-\frac{z^{k-1} g(z)}{\left(z^{N l}-1\right)^{2}},
$$

where $g(z)=\left(k f(z)+z f^{\prime}(z)\right)\left(z^{N l}-1\right)-N l z^{N l} f(z)$. Letting $w(z)=-\left(z^{N l}-1\right)^{2} /\left(z^{k} g(z)\right)$, we obtain

$$
\frac{d r}{d p}=\operatorname{Re}\left(\frac{r}{z} \frac{d z}{d p}\right)=r \operatorname{Re}(w)
$$

We now compute $\operatorname{Re}(w)$. We note that

$$
f(\bar{z})=-\frac{f(z)}{z^{l+2}}, \quad f^{\prime}(\bar{z})=\frac{h(z)}{z^{l}},
$$


where $h(z)=l+1-z^{l}$. From the above equalities and as $z^{M}=1$, we have

$$
\begin{aligned}
\bar{z}^{k} g(\bar{z}) & =\frac{1}{z^{k}}\left\{\left(k f(\bar{z})+\frac{1}{z} f^{\prime}(\bar{z})\right)\left(\frac{1}{z^{N l}}-1\right)-\frac{N l}{z^{N l}} f(\bar{z})\right\} \\
& =\frac{(-k f(z)+z h(z))\left(1-z^{N l}\right)+N l f(z)}{z^{N l+l+2+k}} \\
& =\frac{(-k f(z)+z h(z))\left(1-z^{N l}\right)+N l f(z)}{z^{2 N l-k}} .
\end{aligned}
$$

It follows that

$$
\begin{aligned}
\operatorname{Re}(w) & =\frac{w+\bar{w}}{2} \\
& =-\frac{1}{2}\left\{\frac{\left(z^{N l}-1\right)^{2}}{z^{k} g(z)}+\frac{\left(\bar{z}^{N l}-1\right)^{2}}{\bar{z}^{k} g(\bar{z})}\right\} \\
& =-\frac{1}{2}\left\{\frac{\bar{z}^{k} g(\bar{z})\left(z^{N l}-1\right)^{2}+z^{k} g(z)\left(\bar{z}^{N l}-1\right)^{2}}{|g(z)|^{2}}\right\} \\
& =-\frac{1}{2|g(z)|^{2}}\left\{\frac{(-k f(z)+z h(z))\left(1-z^{N l}\right)+N l f(z)}{z^{2 N l-k}} \cdot\left(z^{N l-1}\right)^{2}\right. \\
& =-\frac{\left(z^{N l}-1\right)^{2} z^{k}}{2 z^{2 N l}|g(z)|^{2}}\left\{(k f(z)-z h(z))\left(z^{N l}-1\right)+N l f(z)\right. \\
& =-\frac{\left(z^{N l}-1\right)^{3} z^{k}}{2 z^{2 N l}|g(z)|^{2}}\left\{2 k f(z)+z\left(f^{\prime}(z)-h(z)\right)-N l f(z)\right\} .
\end{aligned}
$$

Since

$$
2 k f(z)+z\left(f^{\prime}(z)-h(z)\right)-N l f(z)=M f(z),
$$

we obtain

$$
\operatorname{Re}(w)=\frac{\left(z^{N l}-1\right)^{4} M}{2 z^{2 N l}|g(z)|^{2}} \cdot \frac{-z^{k} f(z)}{z^{N l}-1}=\frac{\left(z^{N l}-1\right)^{4} M p}{2 z^{2 N l}|g(z)|^{2}} .
$$

The value of $\operatorname{Re}(w)$ at $z=e^{w_{m} i}$ is

$$
\operatorname{Re}(w)=\frac{\left(z^{N l}-1\right)^{4}}{z^{2 N l}} \cdot \frac{M p}{2|g(z)|^{2}}=\left(2 \cos N l w_{m}-2\right)^{2} \cdot \frac{M p}{2|g(z)|^{2}}>0 .
$$




\section{A note on asymptotic stability conditions}

Therefore,

$$
\frac{d r}{d p}=\frac{2 r\left(\cos N l w_{m}-1\right)^{2} M p}{|g(z)|^{2}}
$$

and it follows that (2.16) holds at $z=e^{w_{m} i}$.

Case $2\left(z^{l}=1\right)$. With an argument similar to Case 1 , we obtain

$$
\frac{d r}{d p}=\frac{2 r N^{2} M p}{|(M+1) z-M+1|^{2}}
$$

which implies that (2.16) is valid for $z=e^{w_{m} i}$.

This completes the proof.

We now determine the minimum of the absolute values of $p_{m}$ given by (2.3). We have the following result.

Proposition 2.4. $\left|p_{0}\right|=\min \left\{\left|p_{m}\right|: m=0,1, \ldots, M-1\right\}$.

To prove Proposition 2.4, we need the following lemma, which was proved in [4]. LEMma 2.5. Let $N$ be a positive integer, then

$$
\left|\frac{\sin N t}{\sin t}\right| \leq N
$$

holds for all $t \in \mathbb{R}$.

Proof of Proposition 2.4. From (2.3), $p_{m}=(-1)^{m+1}\left(\sin \left(l w_{m} / 2\right) / \sin \left(N l w_{m} / 2\right)\right)$. For $m=0$, it follows from L'Hospital's rule that $p_{0}=-1 / N$. For $m=1,2, \ldots, M-1$, we have

$$
\left|p_{m}\right|=\left|(-1)^{m+1} \frac{\sin \left(l w_{m} / 2\right)}{\sin \left(N l w_{m} / 2\right)}\right| \geq \frac{1}{N}
$$

by Lemma 2.5. This completes the proof.

We are now ready to prove Theorem 1.2.

Proof of Theorem 1.2. Note that $F(1)=1+N p \leq 0$ if and only if $p \leq-1 / N$. Since $\lim _{z \rightarrow+\infty} F(z)=+\infty$, it follows that (2.1) has a positive root $\alpha$ such that $\alpha>1$ when $p \leq$ $-1 / N$. We claim that if $|p|$ is sufficiently small, then all the roots of (2.1) are inside the unit disk. To this end, we note that when $p=0,(2.1)$ has exactly one root at 0 of multiplicity $k+1$. By the continuity of the roots with respect to $p$, this implies that our claim is true. By Proposition 2.4, $p_{0}=-1 / N$ and $\left|p_{m}\right| \geq 1 / N$ which implies that $\left|p_{0}\right|=1 / N$ is the smallest positive value of $p$ such that a root of (2.1) intersects the unit circle as $|p|$ increases. Moreover, Proposition 2.3 implies that if $p>p_{\min }$, then there exists a root $\alpha$ of (2.1) such that $|\alpha| \geq 1$, where $p_{\min }$ is the smallest positive real value of $p$ for which (2.1) has a root on $C$. We conclude that all the roots of (2.1) are inside the unit disk if and only if $-1 / N<p<p_{\min }$. In other words, the zero solution of (1.3) is asymptotically stable if and only if condition (1.4) holds. This completes the proof. 


\section{Examples}

Example 3.1. In (1.3), Let $l$ and $k$ be even positive integers, then we have

$$
F(-1)=-1+p N
$$

Thus if $p=1 / N$, then $F(-1)=0$ and we conclude that (1.3) is asymptotically stable if and only if $-1 / N<p<1 / N$.

Example 3.2. In (1.3), let $N=3, l=3$, and $k=6$. Then $M=8$ and we obtain $p_{0}=-1 / 3$, $p_{1}=\sin (3 / 8) \pi / \sin (9 / 8) \pi, p_{2}=-\sin (3 / 4) \pi / \sin (9 / 4) \pi, p_{3}=\sin (9 / 8) \pi / \sin (27 / 8) \pi, p_{4}=$ $-\sin (3 / 2) \pi / \sin (9 / 2) \pi, \quad p_{5}=\sin (15 / 8) \pi / \sin (45 / 8) \pi, \quad p_{6}=-\sin (9 / 4) \pi / \sin (27 / 4) \pi$, and $p_{7}=\sin (21 / 8) \pi / \sin (63 / 8) \pi$. Thus, $p_{3}=p_{5}=\sin (\pi / 8) / \sin (3 \pi / 8)$ is the smallest positive real value of $p$ such that $(2.1)$ has a root on $C$. We conclude that $(1.3)$ is asymptotically stable if and only if $-1 / 3<p<\sin (\pi / 8) / \sin (3 \pi / 8)$.

\section{Acknowledgments}

This research is supported by the Thailand Research Fund Grant no. RTA458005 and RSA4780012. We would like to thank the referees for their valuable comments.

\section{References}

[1] Y. Kuang, Delay Differential Equations with Applications in Population Dynamics, Mathematics in Science and Engineering, vol. 191, Academic Press, Massachusetts, 1993.

[2] S. A. Kuruklis, The asymptotic stability of $x_{n+1}-a x_{n}+b x_{n-k}=0$, J. Math. Anal. Appl. 188 (1994), no. 3, 719-731.

[3] S. A. Levin and R. M. May, A note on difference-delay equations, Theoret. Population Biology 9 (1976), no. 2, 178-187.

[4] R. Ogita, H. Matsunaga, and T. Hara, Asymptotic stability condition for a class of linear delay difference equations of higher order, J. Math. Anal. Appl. 248 (2000), no. 1, 83-96.

[5] V. G. Papanicolaou, On the asymptotic stability of a class of linear difference equations, Math. Mag. 69 (1996), no. 1, 34-43.

[6] G. Stépán, Retarded Dynamical Systems: Stability and Characteristic Functions, Pitman Research Notes in Mathematics Series, vol. 210, Longman Scientific \& Technical, Harlow, 1989.

T. Kaewong: Department of Mathematics, Faculty of Science, Chiang Mai University, Chiang Mai 50200, Thailand

E-mail address: theeradach@tsu.ac.th

Y. Lenbury: Department of Mathematics, Faculty of Science, Mahidol University, 272 Rama 6 Road, Bangkok 10400, Thailand

E-mail address: scylb@mahidol.ac.th

P. Niamsup: Department of Mathematics, Faculty of Science, Chiang Mai University, Chiang Mai 50200, Thailand

E-mail address: scipnmsp@chiangmai.ac.th 


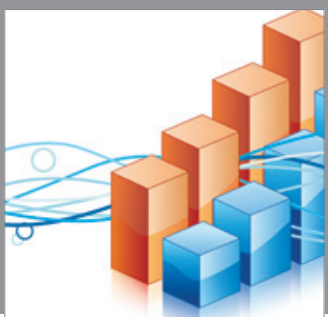

Advances in

Operations Research

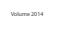

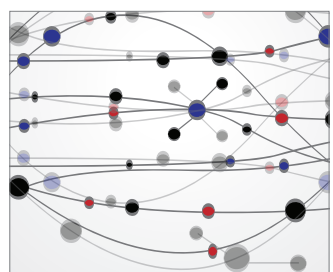

\section{The Scientific} World Journal
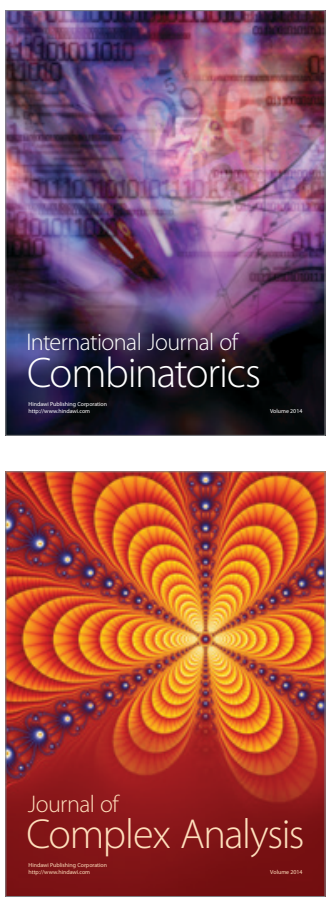

International Journal of

Mathematics and

Mathematical

Sciences
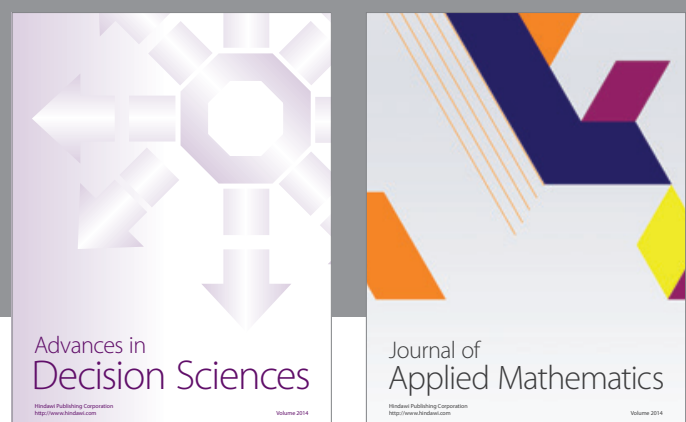

Journal of

Applied Mathematics
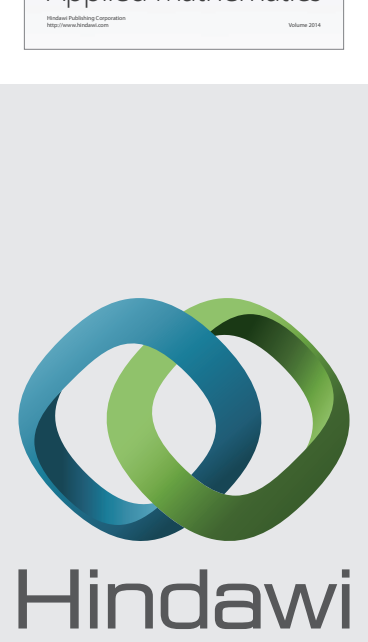

Submit your manuscripts at http://www.hindawi.com
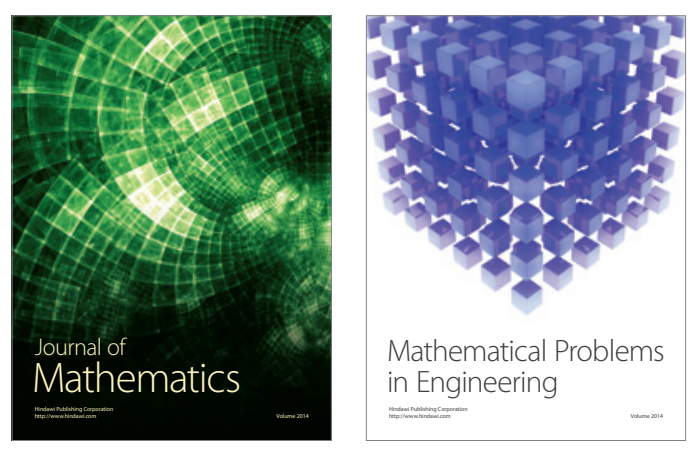

Mathematical Problems in Engineering
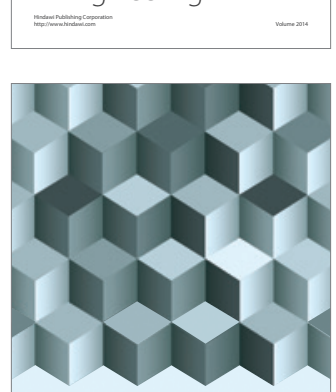

Journal of

Function Spaces
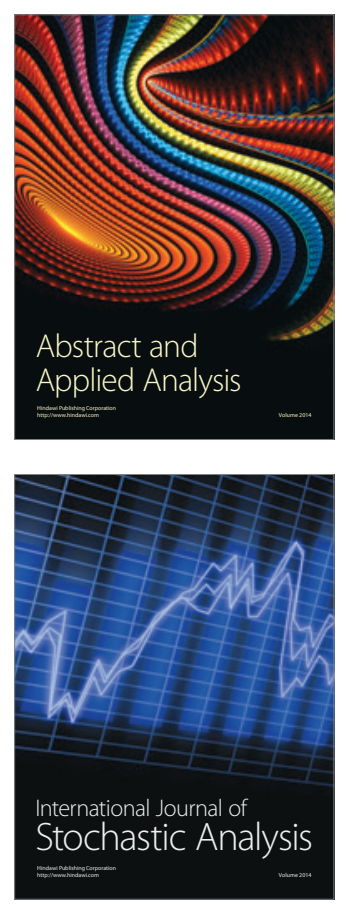

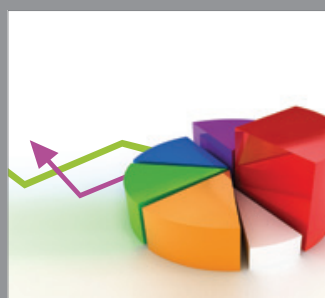

ournal of

Probability and Statistics

Promensencen
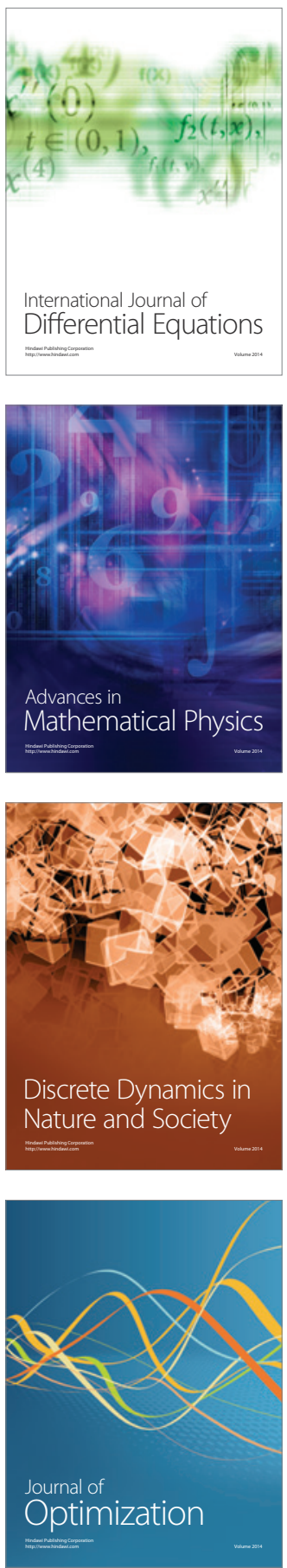